\title{
Experimental Correlation between Nonlinear Optical and Magnetotransport Properties Observed in Au-Co Thin Films
}

\author{
Kaida Yang, ${ }^{1}$ Victor Kryutyanskiy, ${ }^{2}$ Irina Kolmychek, \\ Tatiana V. Murzina, ${ }^{2}$ and R. Alejandra Lukaszew ${ }^{1}$ \\ ${ }^{1}$ Department of Physics, College of William and Mary, Williamsburg, VA 23187, USA \\ ${ }^{2}$ Department of Physics, Moscow State University, Moscow 119992, Russia
}

Correspondence should be addressed to Kaida Yang; kyang@email.wm.edu

Received 15 September 2015; Revised 19 January 2016; Accepted 8 February 2016

Academic Editor: Claude Estournès

Copyright (C) 2016 Kaida Yang et al. This is an open access article distributed under the Creative Commons Attribution License, which permits unrestricted use, distribution, and reproduction in any medium, provided the original work is properly cited.

Magnetic materials where at least one dimension is in the nanometer scale typically exhibit different magnetic, magnetotransport, and magnetooptical properties compared to bulk materials. Composite magnetic thin films where the matrix composition, magnetic cluster size, and overall composite film thickness can be experimentally tailored via adequate processing or growth parameters offer a viable nanoscale platform to investigate possible correlations between nonlinear magnetooptical and magnetotransport properties, since both types of properties are sensitive to the local magnetization landscape. It has been shown that the local magnetization contrast affects the nonlinear magnetooptical properties as well as the magnetotransport properties in magneticmetal/nonmagnetic metal multilayers; thus, nanocomposite films showcase another path to investigate possible correlations between these distinct properties which may prove useful for sensing applications.

\section{Introduction}

Nanocomposite materials where the composition as well as the aggregation state of the different components can be tailored experimentally offer a suitable venue to explore correlation between composition and physical properties that offer greatest benefit for potential applications. On this front, the combination of magnetic metals with noble metals, such as gold or silver, offers a suitable platform to experimentally explore correlations between composition, magnetotransport, and nonlinear magnetooptical properties with an eye towards applications exploiting such correlated properties, particularly in constrained geometries. To this end, Skumryev et al. [1] prepared a class of nanoparticle-matrix materials exhibiting novel exchange bias properties, while Murzina et al. [2] have reported on nonlinear magnetooptical properties of AuCo planar structures on Si and granular AgCo films [3]. The Au-Co system offers an opportunity to experimentally study correlated nonlinear magnetooptical and magnetotransport properties of a nanocomposite thin film.
The Au-Co equilibrium phase diagram reveals simple eutectic behavior with critical eutectic composition and temperature values of 23.5 at. $\% \mathrm{Co}$ in $\mathrm{Au}$ and $996^{\circ} \mathrm{C}$, respectively (Figure 1).

We note that such diagrams represent the bulk behavior of Au-Co binary system, which is often distinct from that of thin films that therefore must be experimentally investigated. Typically, codeposited Au-Co thin films at low $T$ are featureless and smooth, hinting at amorphous structure typical of a homogeneous alloy. It is experimentally observed that subsequent annealing results in segregation of single metastable phases, while further annealing above $650 \mathrm{~K}$ leads to equilibrium phase separation. Such crystallization obeys the so-called Ostwald rule, where a system proceeds from a less stable to a final equilibrium state through a succession of metastable states of increased stability. The amorphous phase is akin to a quenched liquid phase and heat treatment produces transformations from less stable states, with increased Au-Co atomic solubility in amorphous or single-phase metastable matrices, to a two-phase equilibrium 


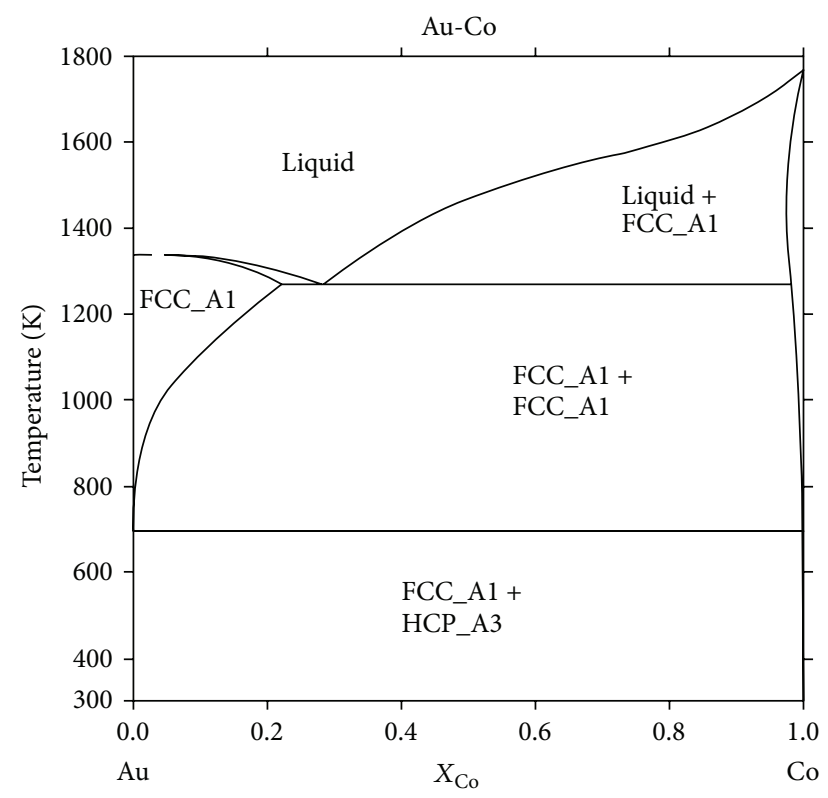

FIGURE 1: Calculated Au-Co phase diagram (corresponding pressure $=1.01325 \times 10^{5} \mathrm{~Pa}$ ) [7].

face-centered cubic (fcc) crystalline phase state. This phase separation has been applied to cosputtered $\mathrm{Co}_{1-x} \mathrm{Au}_{x}$ thin films to engineer their magnetotransport properties after thermal annealing [4]. Thus, since the Au-Co binary system is a typical phase-separation system [5], it enables deposition of granular, nanocomposite films where the Co content as well as Co cluster size can be tailored via adequate deposition and/or processing conditions [6], allowing for experimental exploration of correlations between magnetotransport and nonlinear magnetooptics that could be further exploited in sensing applications.

\section{Experiment}

$\mathrm{Au}-\mathrm{Co}$ nanocomposite thin films were codeposited on glass from high purity (99.99\%) Au and Co targets using DC magnetron sputtering in an ultrahigh vacuum (UHV) chamber with base pressure in the low $10^{-9}$ Torr regime. All the samples in this study had a total thickness of $50 \mathrm{~nm}$ and the Co content varied from $10 \%$ to $58 \%$. The relative composition of $\mathrm{Au}$ and $\mathrm{Co}$ was tailored based upon well-calibrated $\mathrm{Au}$ and Co deposition rates. The deposition temperature was kept at $300^{\circ} \mathrm{C}$ since we observed using Transmission Electron Microscopy (TEM) contrast images along with Electron Energy Loss Spectroscopy (EELS) compositional maps that it was optimal for these studies, leading to adequate nanocomposite and smooth films (Figure 2), while higher deposition temperature led to rougher and more segregated films [4]. All samples were capped with $3 \mathrm{~nm}$ Au films grown at room temperature to prevent Co oxidation after removal from the UHV chamber.

To investigate the experimental correlation between nonlinear optical properties and magnetotransport, we carried out nonlinear magnetooptical (NOMOKE), magnetization reversal, and DC four-point probe magnetotransport studies.
The nonlinear-optical second harmonic generation (SHG) studies were performed using a $\mathrm{Ti}$ : sapphire laser operating at a wavelength of $800 \mathrm{~nm}$ and average power of $200 \mathrm{~mW}$. A $45^{\circ}$ incident beam illuminated the sample and the second harmonic (SH) signal generated in reflection from the films was selected by an appropriate set of filters and detected by a photon counter. The SH polarization configuration is $\mathrm{p}$ in (for the pump beam) and p-out (for the SH beam). A transversal DC magnetic field up to $3 \mathrm{kOe}$ was applied in the plane of the film for an arbitrary azimuthal orientation of the sample, since no in-plane anisotropy in the SHG signal was observed. The NOMOKE magnetic contrast is defined as $\left[I_{2 \omega}(+H)-I_{2 \omega}(-H)\right] /\left[I_{2 \omega}(+H)+I_{2 \omega}(-H)\right]$ with the SHG intensities $I_{2 \omega}( \pm H)$ measured in the opposite directions to the DC magnetic field $\mathrm{H}$.

SHG magnetic hysteresis loops as a function of Co concentration, measured at room temperature, are shown in Figure 3(a). Although there are possible detection limitations for lower Co content with this technique, we still can observe a clear difference in the shape of the hysteresis loops for different Co content. The result suggests evolution from superparamagnetic (isolated magnetic clusters) to ferromagnetic (percolation of the magnetic clusters) behavior as the Co content is increased. The saturation field extracted from the SHG loops is shown in Figure 3(b) where we note a clear decrease in saturation field and the onset of ferromagnetic behavior around $40-45 \%$ Co content. Below the percolation threshold, a Co-Au film can be modeled as a disordered array of spherical metal particles embedded in a homogeneous semi-infinite medium. Film anisotropy and the presence of a second interface bounding the film can be neglected [8]. The transition from ferromagnetic to superparamagnetic magnetic behavior indicates that the nanoparticles with smaller scale size (i.e., lower Co volume concentration) cannot hold the magnetic moment stable. Thus, thermal fluctuations induce the flipping of the magnetic moment. Meanwhile, percolation, possibly generated by the coalescence (direct contact) of magnetic metal phases, can sustain the direction of the magnetic moment when temperature remains unchanged.

Thus, from the SHG saturation field data versus composition (Figures 3(a)-3(b)) we note that the composition of the films corresponds to segregated magnetic nanoclusters embedded in the Au matrix for Co content below $40 \%$, while the decrease observed at $45 \%$ is attributed to percolation of the magnetic clusters accompanied by ferromagnetic behavior.

Magnetization hysteresis $[\mathrm{M}(\mathrm{H})]$ loops were also measured using superconducting quantum interference device (SQUID) magnetometry.

In Figure 4(a), the out-of-plane (easy axis) and in-plane (hard axis) $\mathrm{M}(\mathrm{H})$ loops were measured and we note that the magnetization correlates well with Co concentration and also that the saturation field decreases with increased Co content, indicating that percolation of the magnetic clusters occurs for a Co content around $x=40 \%$, consistent with the NOMOKE data.

Magnetotransport measurements were carried out using the van der Pauw four point probe method [9]. This method 


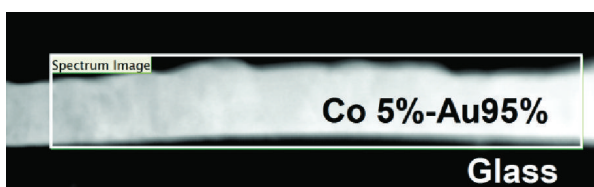

(a)

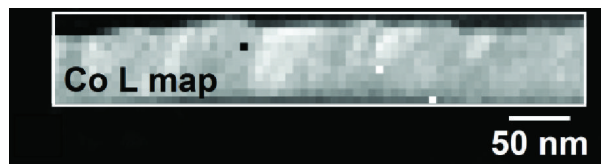

(b)

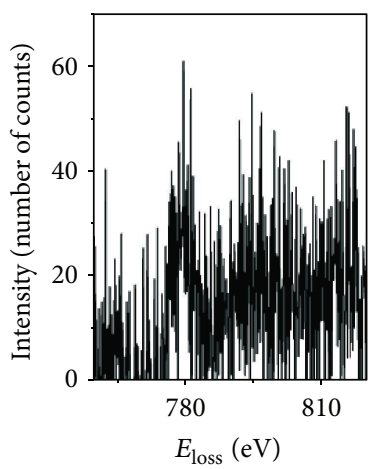

(c)

Figure 2: TEM images (a), EEL spectra (c), and high-resolution composition maps based on EELS/STEM (b) for a typical Au-Co nanocomposite sample. The Co maps were obtained through spectrum imaging after subtracting the background using a power law fit and integrating a window $20 \mathrm{eV}$ wide below the $\mathrm{Co}_{2,3}$ edge around $779 \mathrm{eV}$ (from [6]).

allows measurement of the resistivity of samples with an arbitrary shape via 4 contact points. The magnetoresistance was measured at room temperature with current-in-plane (CIP) configuration, applying an external DC magnetic field parallel to the surface of the samples while current was also applied in the plane of the samples and perpendicular to the external magnetic field. A programmable triggered function was used to minimize measurement errors by recording the corresponding voltage at a preselected time once the current was applied. The magnetoresistance data for $\mathrm{Au}-\mathrm{Co}$ nanocomposite films with varied Co composition is shown in Figure 4(b).

Correlation between the nonlinear magnetooptical and magnetotransport properties in the films can be observed when NOMOKE and magnetoresistance as a function of Co concentration are plotted together in Figure 5. We include selected SHG hysteresis loops as insets in Figure 5 to illustrate the different aggregation states of Co in such samples.

We note that while the NOMOKE signal grows with Co content, following a similar overall trend as the saturation field, a local albeit weak maximum is also observed around $35 \%$ Co content.

\section{Discussion}

Although extensive work has been carried out regarding the magnetotransport properties and magnetooptical properties of Au-Co thin films and multilayers [10,11], to our knowledge no experimental correlated studies between magnetotransport and nonlinear magnetooptical properties in $\mathrm{Au}-\mathrm{Co}$ nanocomposite thin films have yet been presented. We begin our analysis by noting that in such segregated nanocomposite systems, small single-domain magnetic clusters can exist where the sizes of the ferromagnetic domains remain unchanged and hence the external field only rotates the magnetic axes of the clusters towards complete alignment; therefore, the size of the magnetoresistive effect depends on the number and size of magnetic clusters within the probed volume [12]. In our case, the maximum in magnetoresistance near 35\% Co content corresponds to the optimal cluster size (typical grain radius $1.4 \mathrm{~nm}$ ) [10] and intercluster separation for this system [13, 14], consistent with other reports [15]. Low magnetoresistance values for sample composition below $30 \%$ Co content are consistent with a relatively low concentration of magnetic clusters, thus leading to fewer interfacial scattering sites. Increases in Co concentration lead to increased magnetoresistance until an optimal value is reached, beyond which it decreases again due to a reduction of scattering centers since the magnetic clusters have now coalesced and percolated [16-18]. Hence, we have experimental evidence of the role of nanocluster size in the observed properties.

Optical second harmonic generation (SHG) is a sensitive method to probe surface and interface structures because SHG is forbidden in bulk for centrosymmetric materials within the electric dipole approximation. Therefore, the second harmonic light is generated near the surface or interface, where the inversion symmetry is necessarily broken [19]. In the presence of magnetization, the symmetry of the surface and interfaces is further reduced leading to nonlinear magnetooptical effects (NOMOKE). Consequently, the NOMOKE intensity correlates with the relative local magnetization in the material [20-24], that is, at the interface between the Co nanoclusters and the Au matrix in this case. Since our $\mathrm{Au} / \mathrm{Co}$ nanocomposite films do exhibit different interfaces between the magnetic Co clusters and the Au matrix as the Co concentration is increased, we also expect that the ensuing different magnetic coupling will affect the NOMOKE. Indeed, we do observe a slow monotonic increase of NOMOKE with Co concentration, with a sharp increase at the percolation onset around $45 \%$ Co content due to its sensitivity to the aggregation state of the magnetic material, similar to the saturation field in Figure 2(b). We also distinguish an additional subtle regime due to NOMOKE's extreme sensitivity to the local magnetization state, and we observe different behavior related to the local magnetic coupling between Co clusters at a Co content of $35 \%$. Thus, at low Co concentration, the smaller amount of magnetic interfaces leads to slow but steady growth of the NOMOKE signal. With further Co content increase, a local subtle NOMOKE maximum is observed when the local magnetic contrast is large, as it is 

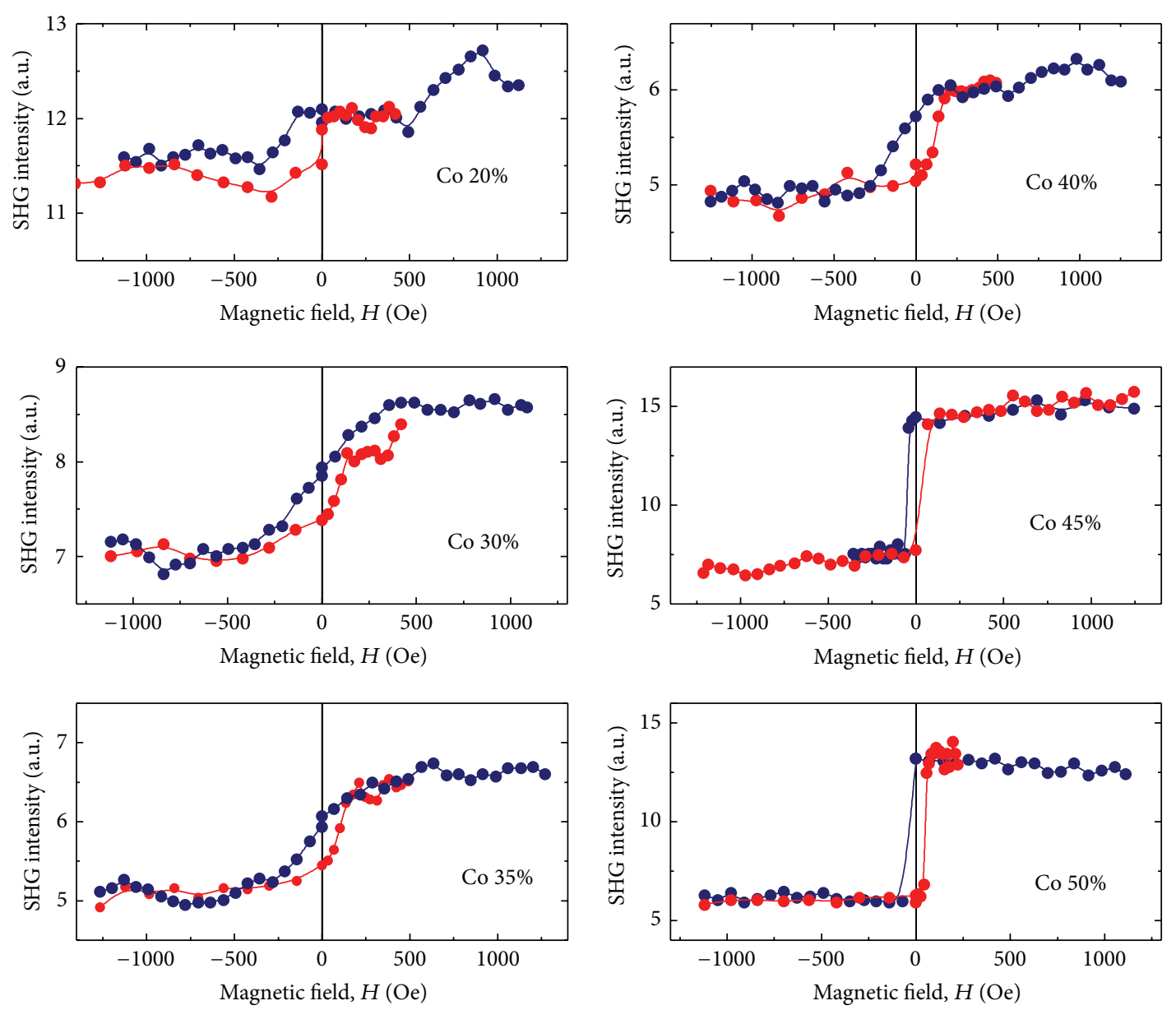

(a)

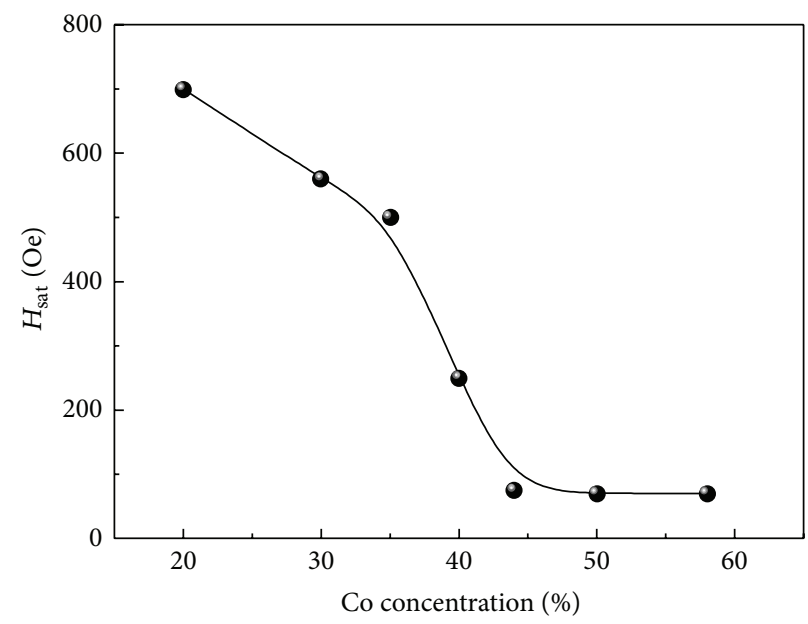

(b)

Figure 3: (a) SHG magnetic hysteresis loops as a function of Co concentration. (b) The corresponding saturation field obtained from the magnetic hysteresis loops as a function of Co content. 


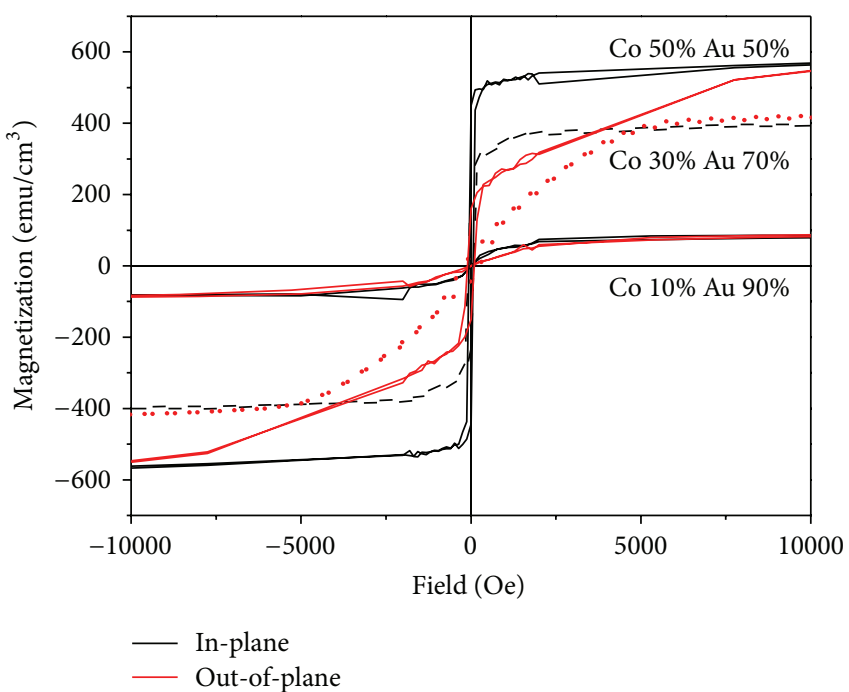

(a)

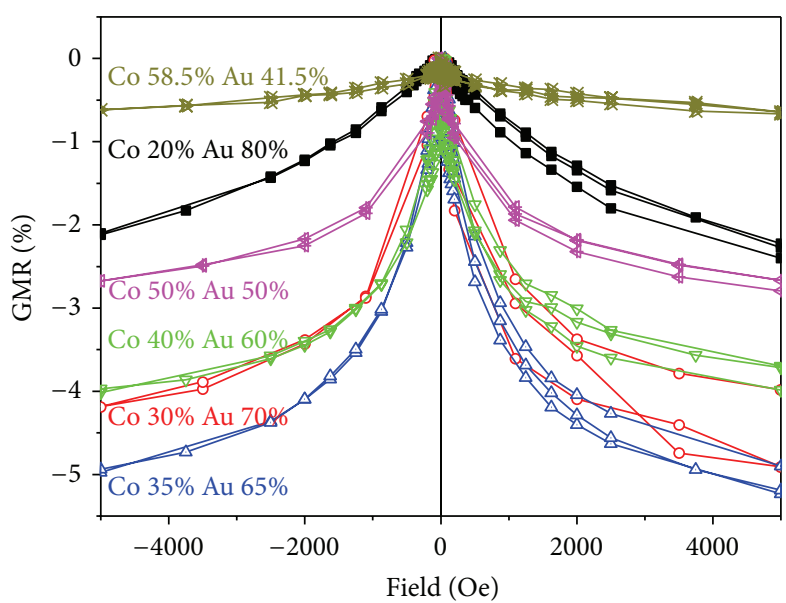

(b)

FIGURE 4: (a) Hysteresis loops obtained using SQUID magnetometry for selected nanocomposite films with different Co concentrations. (b) Room temperature magnetoresistance versus applied magnetic field for nanocomposite thin films with the composition indicated.

antiferromagnetic coupling [25] coincident with the large magnetoresistance observed for this Co content. A strong NOMOKE change occurs as soon as percolation sets in for larger Co concentration transitioning to the steady growth observed at lower Co concentrations.

In summary, since the coupling between Co nanoclusters depends on their size and separation, which is in turn related to the Co concentration, we experimentally observe that, at $35 \% \mathrm{Co}$, our films achieved adequate size and intergranular Co cluster separation, possibly leading to local antiferromagnetic coupling responsible for larger magnetoresistance as well as enhanced local magnetic contrast and hence a subtle local maximum in NOMOKE. Our experimental work opens up the opportunity to explore the theoretical correlation between the microscopic hyperpolarizabilities, the second order susceptibilities described by the third rank polar tensors, hyper Rayleigh scattering, and quadruple contributions as well as the role of spin orientation and interactions with phonons [26-30].

The interactions of Co nanocomposite films consisting of Co particles and different matrices have been the subject of many studies [28-33]. When the conductive Au matrix is replaced with other nonconductive materials, for example, $\mathrm{TiO}_{2}[28,29]$ and $\mathrm{Sm}_{2} \mathrm{O}_{3}$, the magnetoresistance dependence on Co concentration in a similar granular thin film structure also shows strong correlation with magnetooptical properties. It should be pointed out that the characteristics of the magnetooptical properties, that is, nonmonotonic response dependency on concentration, is quite similar to that of magnetotransport properties in various Co-matrix systems. The observed similar behavior in these systems shows bulklike behavior, very likely due to Co nanoparticle percolation dominating the magnetotransport and magnetooptical response.

\section{Conclusion}

The microstructure and composition of granular and nanocomposite AuCo thin film samples can be experimentally tailored with adequate choice of deposition and/or processing conditions since Au-Co is a typical phase-separation system. We have experimentally observed that the aggregation state of the films affects their nonlinear magnetooptical properties as well as their magnetotransport behavior in a subtle, correlated manner. The NOMOKE values are affected by contrast at the local cluster/matrix interface in nanocomposite films, while the magnetotransport properties primarily depend on the magnetic coupling between magnetic clusters. The observed correlation between the SHG magnetic contrast and magnetoresistance below percolation may be expected in these nanocomposite films, considering that the cluster separation determines the magnetic coupling and hence the spin-dependent electron scattering. NOMOKE is driven by the local magnetic contrast, also affected by the magnetic cluster size and intercluster distance. On the other hand, above the percolation threshold, the behavior of NOMOKE and that of the magnetoresistance are quite different because the increase in magnetization is responsible for the observed monotonic increase in NOMOKE due to the presence of more magnetic materials, although the ensuing ferromagnetic order suppresses spin-dependent electron scattering and thus decreases the magnetoresistance. The detailed nature of this correlation must be further investigated theoretically, perhaps combining approaches previously developed for each of these separate phenomena in order to provide a quantitative model for the Co content dependence in our reported experimental magnetoresistance and NOMOKE data. Further, theoretical correlation between the microscopic hyperpolarizabilities, the second order 


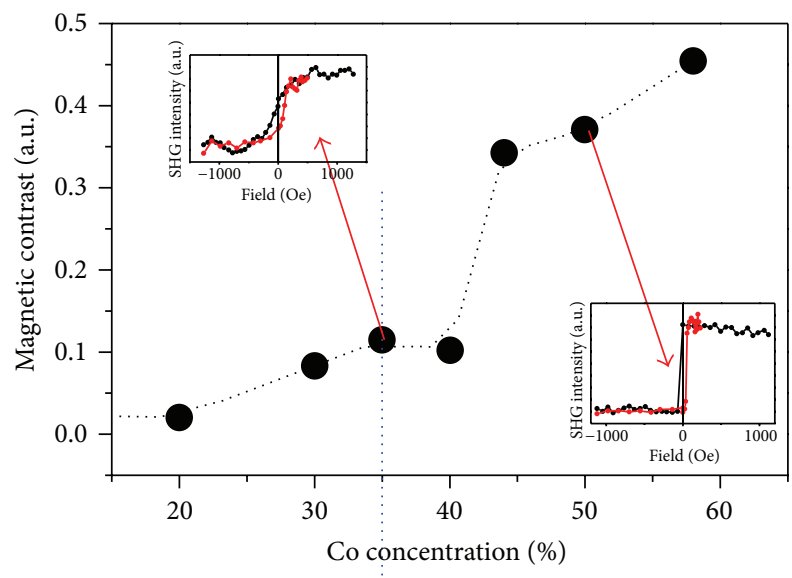

(a)

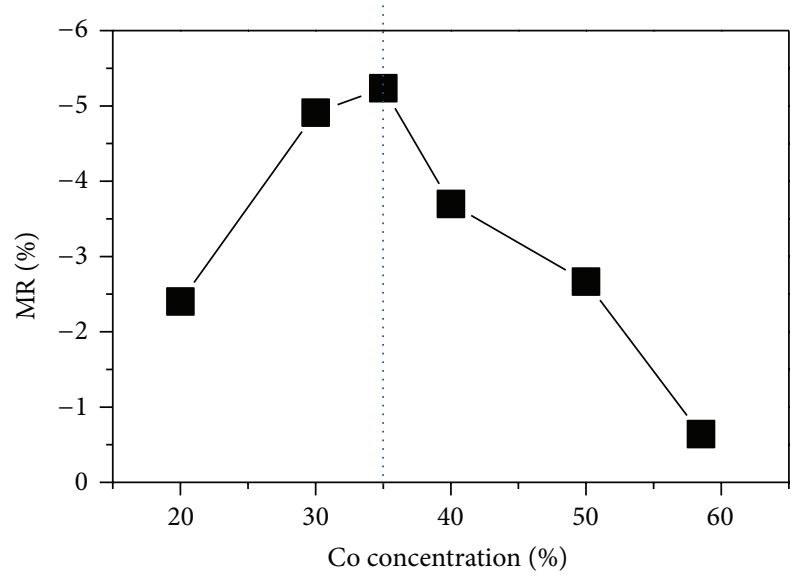

(b)

FIGURE 5: SHG magnetic contrast (a) and magnetoresistance (b) versus $\mathrm{Co}$ content. The dashed line indicates that alocal weak maximum in the NOMOKE signal correlates with the magnetoresistance (MR) maximum. Inset. SHG hysteresis loops for selected samples.

susceptibilities described by the third rank polar tensors, hyper Rayleigh scattering, and quadruple contributions, as well as the role of the spin orientation and interactions with phonons could be investigated. Yet our experimental findings are useful for applications such as double-sensors where two different sensing properties, that is, magnetotransport and magnetooptics, could be addressed simultaneously.

\section{Conflict of Interests}

The authors declare that there is no conflict of interests regarding the publication of this paper.

\section{Acknowledgment}

Aspects of this work have been supported by the Virginia Microelectronics Consortium (VMEC) and by the US Department of Energy (Award DE-SC0008546).

\section{References}

[1] V. Skumryev, S. Stoyanov, Y. Zhang, G. Hadjipanayis, D. Givord, and J. Nogués, "Beating the superparamagnetic limit with exchange bias," Nature, vol. 423, no. 6942, pp. 850-853, 2003.

[2] V. Murzina, A. V. Sherbarshin, A. I. Klomychek et al., "Magnetism of a cobalt-gold planar nanos-tructure on the silicon surface," Journal of Experimental and Theoretical Physics, vol. 136, pp. 123-134, 2009.

[3] T. V. Murzina, T. V. Misuryaev, A. A. Nikulin, O. A. Aktsipetrov, and J. Güdde, "Correlation between GMR and nonlinear magneto-optical Kerr effect in granular films," Journal of Magnetism and Magnetic Materials, vol. 258-259, pp. 99-102, 2003.

[4] H. S. Nalwa, Ed., Handbook of Surfaces and Interfaces of Materials, vol. 1 of Surface and Interface Phenomena, 2001.

[5] H. Okamoto and T. B. Massalski, Phase Diagrams of Binary Gold Alloys, ASM International, Materials Park, Ohio, USA, 1987.

[6] K. Yang, C. Clavero, J. R. Skuza, M. Varela, and R. A. Lukaszew, "Surface plasmon resonance and magneto-optical enhancement on Au-Co nanocomposite thin films," Journal of Applied Physics, vol. 107, no. 10, Article ID 103924, 2010.

[7] http://resource.npl.co.uk/mtdata/phdiagrams/auco.htm.

[8] T. V. Murzina, T. V. Misuryaev, A. F. Kravets et al., "Nonlinear magneto-optical Kerr effect and plasmon-assisted SHG in magnetic nanomaterials exhibiting giant magnetoresistance," Surface Science, vol. 482-485, no. 2, pp. 1101-1106, 2001.

[9] L. J. van der Pauw, Philips Research Reports, vol. 13, Eindhoven University of Technology, Eindhoven, The Netherlands, 1958.

[10] G. Verschoren, A. N. Dobrynin, K. Temst et al., "Electron scattering in Au films containing Co clusters," Thin Solid Films, vol. 516, no. 23, pp. 8232-8239, 2008.

[11] C. Christides, "Magnetotransport properties of ultrathin metallic multilayers: microstructural modifications leading to sensor applications," in Handbook of Surfaces and Interfaces of Materials, H. S. Nalwa, Ed., vol. 4 of Solid Thin Films and Layers, chapter 2, Academic Press, New York, NY, USA, 2001.

[12] J. Q. Xiao, J. S. Jiang, and C. L. Chien, "Giant magnetoresistance in nonmultilayer magnetic systems," Physical Review Letters, vol. 68, no. 25, pp. 3749-3752, 1992.

[13] M. Pan, H. Liu, J. Wang et al., "Quantum growth of magnetic nanoplatelets of Co on Si with high blocking temperature," Nano Letters, vol. 5, no. 1, pp. 87-90, 2005.

[14] C. L. S. Rizal, A. Yamada, Y. Hori, S. Ishida, M. Matsuda, and Y. Ueda, "Magnetic properties and magnetoresistance effect in $\mathrm{Co} / \mathrm{Au}, \mathrm{Ag}$ nano-structure films produced by pulse electrodeposition," Physica Status Solidi (C), vol. 1, no. 7, pp. 1756-1759, 2004.

[15] C. Rizal, Y. Ueda, and R. K. Pokharel, "Magnetotransport properties of Co-Au granular alloys," International Journal of Applied Physics and Mathematics, vol. 1, no. 3, pp. 161-166, 2011.

[16] I. Inoue, A. Oguri, and S. Maekawa, "Theory of giant magnetoresistance in metallic superlattices," Journal of the Physical Society of Japan, vol. 60, pp. 376-385, 1991.

[17] R. F. Soohoo, Magnetic Thin Films, Harper \& Row, New York, NY, USA, 1965.

[18] R. M. Bozorth, Ferromagnetism, Van Nostrand Reinhold Company, New York, NY, USA, 1947.

[19] R. V. Pisarev, B. B. Krichevtsov, V. N. Gridnev, V. P. Klin, D. Frohlich, and C. Pahlke-Lerch, "Optical second-harmonic generation in magnetic garnet thin films," Journal of Physics: Condensed Matter, vol. 5, no. 45, pp. 8621-8628, 1993. 
[20] G. Tessier, C. Malouin, P. Georges et al., "Magnetizationinduced second-harmonic generation enhanced by surface plasmons in ultrathin Au/Co/Au metallic films," Applied Physics B: Lasers and Optics, vol. 68, no. 2-3, pp. 545-548, 1999.

[21] V. V. Pavlov, G. Tessier, C. Malouin et al., "Observation of magneto-optical second-harmonic generation with surface plasmon excitation in ultrathin $\mathrm{Au} / \mathrm{Co} / \mathrm{Au}$ films," Applied Physics Letters, vol. 75, no. 2, pp. 190-192, 1999.

[22] W. Hübner and K. Bennemann, "Nonlinear magneto-optical Kerr effect on a nickel surface," Physical Review B, vol. 40, article 5973, 1989.

[23] S. Zhang and P. M. Levy, "Conductivity and magnetoresistance in magnetic granular films (invited)," Journal of Applied Physics, vol. 73, no. 10, pp. 5315-5319, 1993.

[24] Yu. G. Pogorelov, M. M. P. de Azevedo, and J. B. Sousa, "Microscopic theory of magnetoresistance in granular materials," Physical Review B, vol. 58, no. 1, pp. 425-431, 1998.

[25] M. Kitada, K. Yamamoto, and N. Shimizu, "Magnetoresistance of co-sputtered Ag-Co-Ni alloy thin films," Journal of Magnetism and Magnetic Materials, vol. 124, no. 3, pp. 243-245, 1993.

[26] K. D. Singer, M. G. Kuzyk, and J. E. Sohn, "Second-order nonlinear-optical processes in orientationally ordered materials: relationship between molecular and macroscopic properties," Journal of the Optical Society of America B, vol. 4, no. 6, pp. 968-976, 1987.

[27] I. V. Kityk, J. Ebothé, I. Fuks-Janczarek et al., "Nonlinear optical properties of Au nanoparticles on indium-tin oxide substrate," Nanotechnology, vol. 16, no. 9, pp. 1687-1692, 2005.

[28] D. B. Hollis, "Review of hyper-Rayleigh and second-harmonic scattering in minerals and other inorganic solids " American Mineralogist, vol. 73, pp. 701-706, 1988.

[29] E. C. Hao, G. C. Schatz, R. C. Johnson, and J. T. Hupp, "HyperRayleigh scattering from silver nanoparticles," The Journal of Chemical Physics, vol. 117, no. 13, pp. 5963-5966, 2002.

[30] Y. Song and H. Dery, "Analysis of phonon-induced spin relaxation processes in silicon," Physical Review B, vol. 86, no. 8, Article ID 085201, 2012.

[31] E. A. Gan'shina, M. Y. Kochneva, P. N. Scherbak, K. Aimuta, and M. Inoue, "Magneto-optical properties of Co-based nanocomposites," in Proceedings of the Digests of the IEEE International Magnetics Conference (INTERMAG Asia '05), pp. 2077-2078, IEEE, Nagoya, Japan, April 2005.

[32] E. Gan’shina, M. Kochneva, M. Vashuk et al., "Magneto-optical properties of magnetic nanocomposites," The Physics of Metals and Metallography, vol. 102, supplement 1, pp. S32-S35, 2006.

[33] Y. Okamoto, K. Nagata, T. Adachi, T. Imanaka, K. Inamura, and T. Takyu, "Preparation and characterization of highly dispersed cobalt oxide and sulfide catalysts supported on $\mathrm{SiO}_{2}$," Journal of Physical Chemistry, vol. 95, no. 1, pp. 310-319, 1991. 

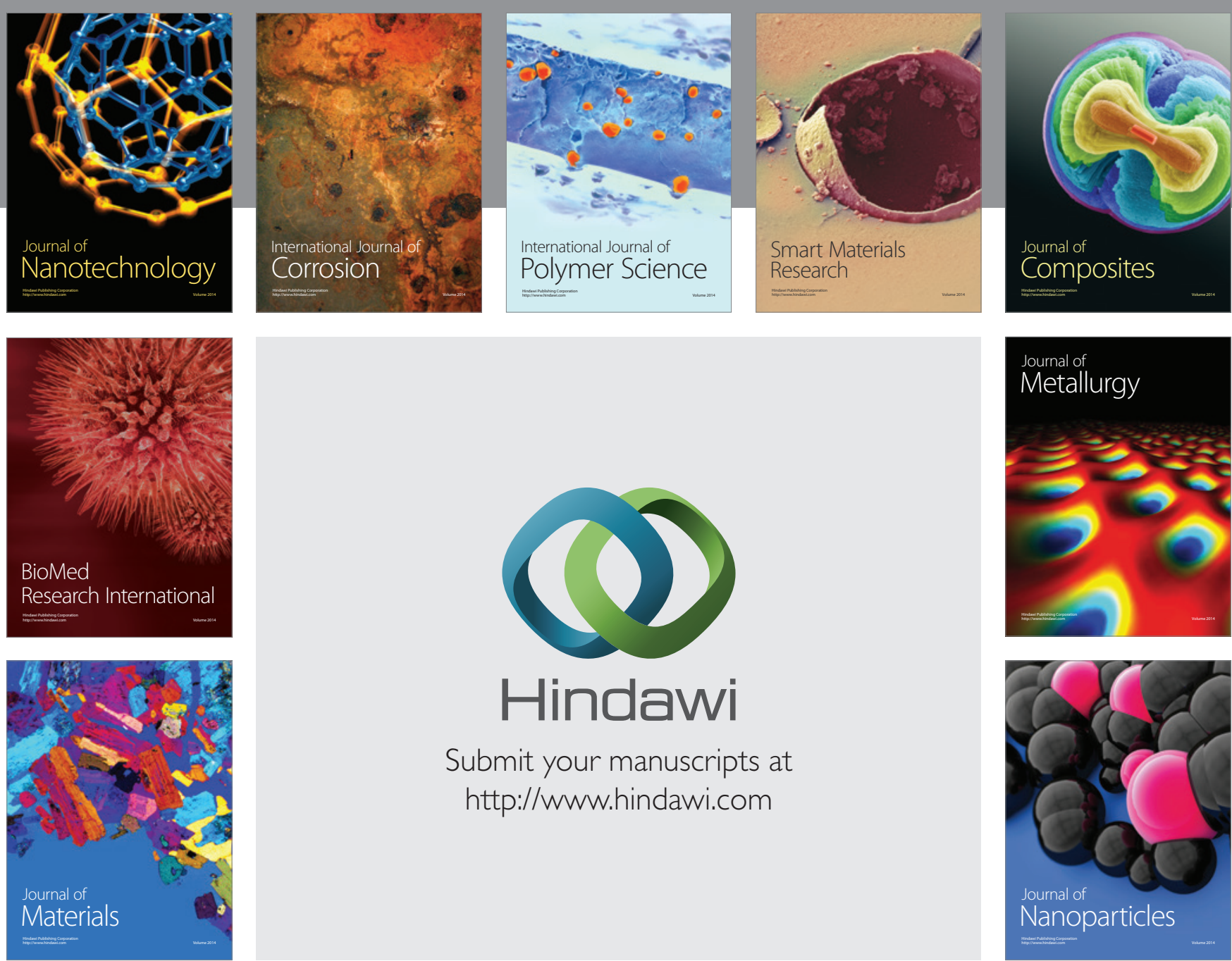

\section{Hindawi}

Submit your manuscripts at

http://www.hindawi.com

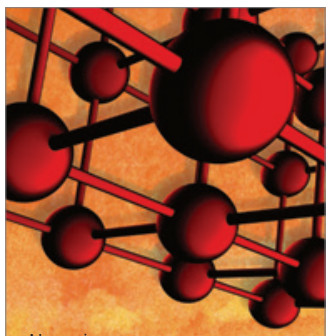

Materials Science and Engineering
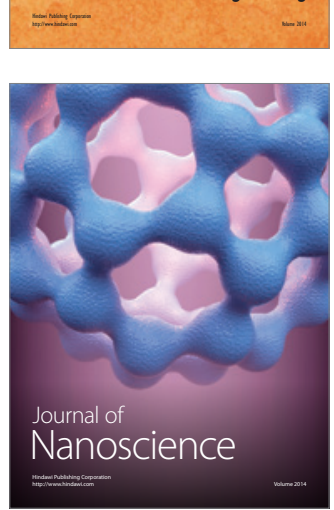
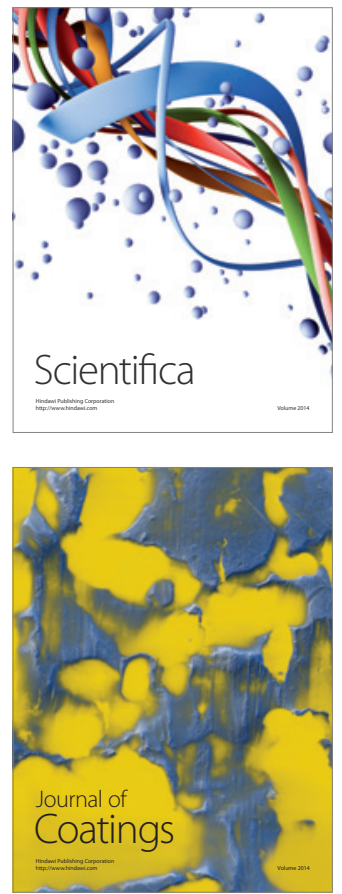
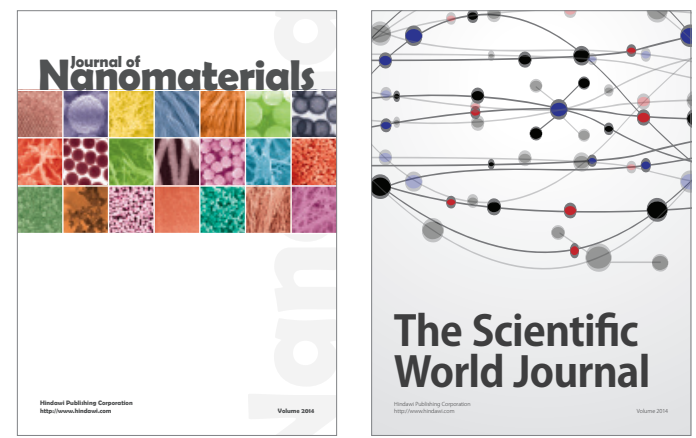

The Scientific World Journal
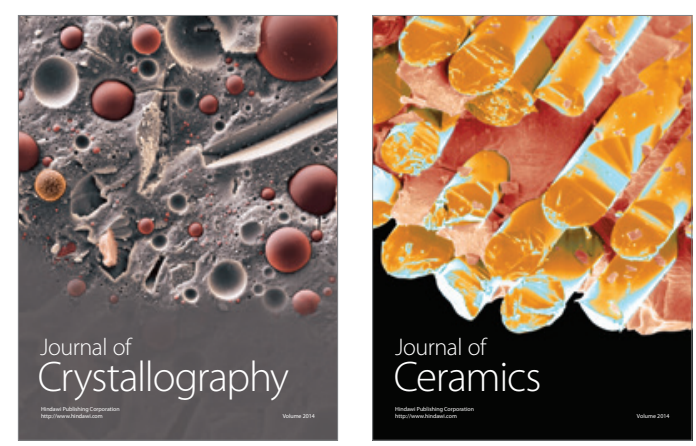
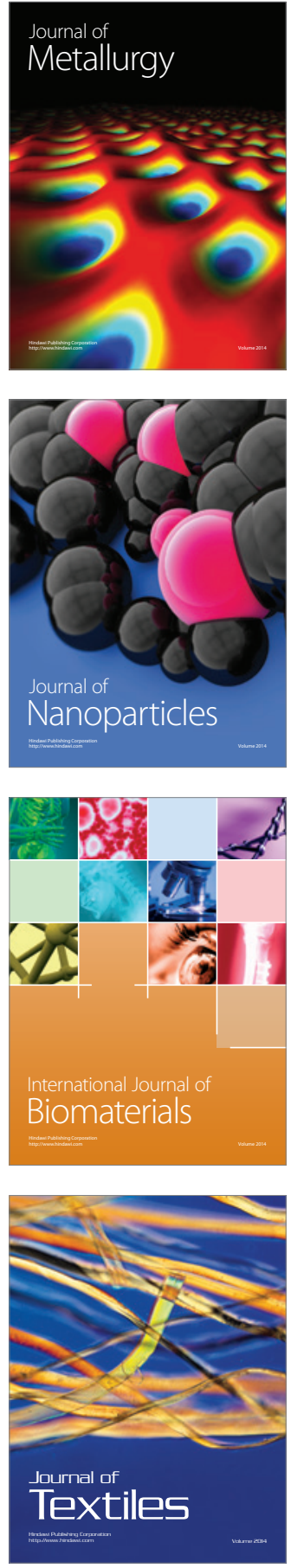